\title{
Oxidative damage associated with thermal stress in Pocillopora verrucosa from the Mexican Pacific
}

\section{Daño oxidativo asociado al estrés térmico en Pocillopora verrucosa en el Pacífico mexicano}

\author{
Alma Paola Rodríguez-Troncoso ${ }^{1}$, Eugenio Carpizo-Ituarte ${ }^{2 *}$, Amilcar Cupul-Magaña ${ }^{1}$ \\ ${ }^{1}$ Centro Universitario de la Costa, Universidad de Guadalajara, Av. Universidad No. 203, Puerto Vallarta, \\ CP 48280, Jalisco, México. \\ 2 Instituto de Investigaciones Oceanológicas, Universidad Autónoma de Baja California, Km 103 Carretera \\ Ensenada-Tijuana, Ensenada, CP 22860, Baja California, México.
}

* Corresponding author: ecarpizo@uabc.edu.mx; ecarpizo@gmail.com

\begin{abstract}
ASBTRACT. Coral communities are under increasing pressure from anomalous increases in sea surface temperature. These events have had negative effects on reef ecosystems and it is important to get a better understanding of the cellular mechanisms that allow organisms to survive the stress period before bleaching is evident. To gain insight into the effect of increased temperature on coral communities off the Pacific coast of central Mexico, we evaluated CuZnSOD (superoxide dismutase) activity in branches of Pocillopora verrucosa by simulating stress conditions $\left(31^{\circ} \mathrm{C}\right.$ ) and non-stress conditions. There was an increase in SOD activity after $18 \mathrm{~h}$ of exposing the corals to $31^{\circ} \mathrm{C}$, while no increase in activity was observed at $28^{\circ} \mathrm{C}$. These results were used to quantify the maximum levels of CuZnSOD activity before Symbiodinium were expelled to prevent cell damage due to oxidative stress. We determined that CuZnSOD can be used as a molecular marker to quantify stress levels, prior to bleaching, in coral communities of the region.
\end{abstract}

Key words: biomarker, coral community, oxidative stress, host.

RESUMEN. La presión ejercida por condiciones de incrementos anómalos en la temperatura superficial del mar sobre las comunidades arrecifales cada vez se presenta con mayor intensidad y frecuencia. Dichos eventos han tenido efectos negativos sobre estos ecosistemas, y es por esto que hay una urgente necesidad de comprender los mecanismos celulares que le permiten al organismo sobrevivir durante el periodo de estrés antes de que el blanqueamiento sea evidente. Para tener un primer conocimiento del efecto del incremento en la temperatura sobre los corales del Pacifico central mexicano, se evaluó la actividad de la CuZnSOD en fragmentos de Pocillopora verrucosa sometidos a condiciones de estrés experimental $\left(31^{\circ} \mathrm{C}\right)$ y a condiciones sin estrés. Los resultados mostraron un incremento en la actividad de la CuZnSOD desde las $18 \mathrm{~h}$ de exposición al estrés $\left(31^{\circ} \mathrm{C}\right)$, mientras que no se observó un incremento en la actividad a los $28^{\circ} \mathrm{C}$. Dichos resultados permitieron evaluar los niveles máximos de actividad de la CuZnSOD que puede mantener el hospedero previo a que expulse al endosimbionte (género Symbiodinium) como acción de defensa para evitar daños celulares asociados al estrés oxidativo. Con los resultados encontrados se puede establecer que la CuZnSOD puede ser utilizada como un marcador celular para evaluar los niveles de estrés, previo al blanqueamiento, en las comunidades coralinas de la región.

Palabras clave: biomarcador, comunidad coralina, estrés oxidativo, hospedero.

\section{INTRODUCTION}

Coral ecosystems are under increasing pressure and stress from human activities and climate change, and are being threatened worldwide (Veron et al. 2009). An anomalous increase in temperature has a negative impact on hermatypic corals and causes bleaching (Brown 1997, Hoegh-Guldberg 1999, Hoegh-Guldberg et al. 2007). Since coral bleaching has occurred with greater intensity and frequency in recent years (Kleypas et al. 2001, Hoegh-Guldberg et al. 2007), efforts have been made to understand the physiological effects of thermal stress (Van Oppen and Gates 2006, Oliver and Palumbi 2011) and how they will affect the continuity of coral communities at the regional level.

\section{INTRODUCCIÓN}

Actualmente, la presión y el estrés sobre los ecosistemas arrecifales se están intensificando por el impacto antropogénico y el cambio climático, los cuales tienen una influencia en todo el planeta y se espera que produzcan un grave deterioro de estos ecosistemas (Veron et al. 2009). Entre las consecuencias de estos cambios, se produce el incremento anómalo en la temperatura, lo cual genera un impacto negativo sobre los corales hermatípicos, produciendo el blanqueamiento (Brown 1997, Hoegh-Guldberg 1999, HoeghGuldberg et al. 2007). Debido a que el blanqueamiento de corales se ha presentado con mayor intensidad y frecuencia en los últimos años (Kleypas et al. 2001, Hoegh-Guldberg 
In the last decades, stress-induced cellular responses have been studied to examine organisms before bleaching becomes evident and assess the health of coral reefs (Gates and Edmunds 1999, Downs et al. 2000). Anomalous increases in temperature cause increased production of oxygen reactive species (ROS) (Fitt et al. 2001, Lesser 2006). The overproduction of ROS leads to oxidative stress in the coral because the amount of $\mathrm{O}^{2-}$ radicals increases; this affects the functionality of the cell and, in response, the host expels its endosymbionts (Downs et al. 2002, Weis 2008).

The enzyme superoxide dismutase (SOD) plays a primary role in the defense against oxidative stress (Zelko et al. 2002). Three isoforms of the antioxidant SOD have been characterized, but CuZnSOD activity has only been observed in the host (Richier et al. 2003, Furla et al. 2005). Thus, by assessing the changes in the levels of CuZnSOD activity, a correlation can be established between the activity of this enzyme and the host's ability to tolerate oxidative stress caused by endosymbiotic dinoflagellates (Symbiodinium) in response to an increase in temperature. Under stress, corals expel their endosymbionts to prevent cell damage and bleaching occurs. The findings will contribute to understand the effects of anomalous increases in sea surface temperature (e.g., El Niño/Southern Oscillation) and the long-term effect of climate change on coral communities in the Mexican Pacific.

\section{MATERIAL AND METHODS}

\section{Thermal stress experiment}

Fragments of the coral Pocillopora verrucosa were collected off Redonda Island in the Marietas National Park $\left(20^{\circ} 40^{\prime} 35^{\prime \prime}-20^{\circ} 41^{\prime} 45^{\prime \prime} \mathrm{N}, 105^{\circ} 33^{\prime} 30^{\prime \prime}-105^{\circ} 38^{\prime} 10^{\prime \prime} \mathrm{W}\right)$, on the Pacific coast of central Mexico. The samples were transported to the laboratory and placed in six tanks. They were allowed to acclimate for 10 days at $27^{\circ} \mathrm{C}$ and then exposed to thermal stress. The experimental design consisted of two treatments, in triplicate, one at $28^{\circ} \mathrm{C}$ (non-stressful temperature) and the other at $31^{\circ} \mathrm{C}$ (temperature associated with bleaching). The temperature was increased gradually during 6 and $8 \mathrm{~h}$, respectively. The experiment lasted $198 \mathrm{~h}$, until bleaching occurred.

During the acclimation and experimental periods, temperature, salinity, and dissolved oxygen were recorded hourly. Dissolved oxygen $\left(\mathrm{mg} \mathrm{L}^{-1}\right)$ was measured using a multiparameter meter (YSI 85). A thermograph (HOBO Water Temp Pro) was placed in each tank to record water temperature every $15 \mathrm{~min}$.

Conditions in the tanks were kept constant during the experiment. In the $28^{\circ} \mathrm{C}$ treatment, temperature ranged from 27.74 to $28.52{ }^{\circ} \mathrm{C}$ (mean \pm standard error of $28.33 \pm 0.04{ }^{\circ} \mathrm{C}$ ), salinity was $35.18 \pm 0.16$, and dissolved oxygen, $5.88 \pm 0.07$ $\mathrm{mg} \mathrm{L}{ }^{-1}$. In the $31^{\circ} \mathrm{C}$ treatment, temperature ranged from 30.6 to $31.02{ }^{\circ} \mathrm{C}$, salinity was $35.24 \pm 0.21$, and dissolved oxygen, $5.78 \pm 0.08 \mathrm{mg} \mathrm{L}^{-1}$. et al. 2007), se han generado grandes esfuerzos para comprender las consecuencias fisiológicas ante las condiciones de estrés térmico (Van Oppen y Gates 2006, Oliver y Palumbi 2011) y cómo éstas afectarán la permanencia de las comunidades coralinas a nivel regional.

En las últimas décadas se han estudiado las respuestas celulares que se desencadenan por efecto de estrés para evaluar al organismo antes de que sea evidente el blanqueamiento (Gates y Edmunds 1999, Downs et al. 2000) y poder evaluar el estado de salud de las poblaciones. Ante incrementos anómalos en la temperatura, se produce una mayor cantidad de especies reactivas de oxígeno (ROS, por sus siglas en inglés) (Fitt et al. 2001, Lesser 2006). Esta sobreproducción de ROS genera estrés oxidativo en el coral al incrementar la cantidad de radicales $\mathrm{O}^{2-}$; esto compromete la funcionalidad de la célula y, como respuesta, el hospedero expulsa al endosimbionte (Downs et al. 2002, Weis 2008).

Se conoce que la superóxido dismutasa (SOD) forma parte de la primera respuesta de defensa enzimática por estrés oxidativo (Zelko et al. 2002). Se han caracterizado tres isoformas del antioxidante SOD; sin embargo, se ha encontrado que la CuZnSOD tiene actividad exclusivamente en el hospedero (Richier et al. 2003, Furla et al. 2005). Por lo tanto, la evaluación de los cambios en los niveles de actividad de la CuZnSOD permitirán establecer una correlación entre la actividad de esta enzima y la capacidad de tolerancia del hospedero ante el estrés oxidativo causado por el endosimbionte (género Symbiodinium) en respuesta a un incremento en la temperatura. El estrés oxidativo se manifiesta con las expulsión del endosimbionte y se visualiza con el blanqueamiento del tejido del coral. Estos resultados permitirán generar aproximaciones celulares del efecto que tienen los eventos de incrementos anómalos en la temperatura superficial del mar, como el fenómeno El Niño/Oscilación del Sur (ENOS), y del efecto a largo plazo del cambio climático en las comunidades coralinas del Pacífico mexicano.

\section{MATERIALES Y MÉTODOS}

\section{Experimento de estrés térmico}

Los fragmentos del coral Pocillopora verrucosa fueron recolectados en la isla Redonda en el Parque Nacional Islas Marietas $\left(20^{\circ} 40^{\prime} 35^{\prime \prime}\right.$ y $20^{\circ} 41^{\prime} 45^{\prime \prime} \mathrm{N}, 105^{\circ} 33^{\prime} 30^{\prime \prime}$ y $105^{\circ} 38^{\prime} 10^{\prime \prime} \mathrm{O}$ ) en el Pacifico central mexicano. Las muestras fueron transportadas al laboratorio y colocadas en 6 acuarios, donde fueron aclimatadas durante 10 días a una temperatura de $27^{\circ} \mathrm{C}$. Posteriormente, los fragmentos fueron expuestos a condiciones de estrés térmico experimental. El diseño experimental consistió en dos tratamientos con tres repeticiones a $28^{\circ} \mathrm{C}$ (temperatura registrada como no estresante) y a $31{ }^{\circ} \mathrm{C}$ (temperatura registrada como disparadora de blanqueamiento). El aumento de la temperatura se realizó de manera gradual durante 6 y $8 \mathrm{~h}$, respectivamente. El experimento 


\section{Quantification of CuZnSOD activity}

The activity of CuZnSOD was determined using the Bioxytech SOD-525 kit (Oxis International, Inc.). To obtain tissue, samples preserved in RNAlater were macerated in $2.0 \mathrm{~mL}$ of a $0.9 \% \mathrm{NaCl}$ solution. Then, $250 \mu \mathrm{L}$ of the homogenate were transferred to a clean tube and $400 \mu \mathrm{L}$ of an ethanol:chloroform solution $(6.25: 37.50, \mathrm{v} / \mathrm{v})$ were added. The sample was mixed using a vortex mixer and centrifuged at $3000 \mathrm{~g}$ for $5 \mathrm{~min}$ at $4{ }^{\circ} \mathrm{C}$. After 3-5 min, the upper liquid part was transferred to a clean $2.0-\mathrm{mL}$ tube. As this process deactivates Mn- and Fe-SOD, just the activity of CuZnSOD can be evaluated.

The extract was treated using the Bioxytech SOD-525 kit according to the manufacturer's instructions, and absorbance at $525 \mathrm{~nm}$ was measured using a Bio-Rad SmartSpec 3000 spectrophotometer. Four readings were made to standardize the blank following the manufacturer's instructions and the samples were read in duplicate. Enzyme activity was expressed in units per milliliter $\left(\mathrm{U} \mathrm{mL}^{-1} \pm\right.$ standard error).

Statistical analyses were performed using Sigma Plot v.11.0 for Windows. Since data did not meet the assumption of normality, the Kruskal-Wallis test (one-way analysis of variance by ranks) was used to determine the effect of temperature and time of exposure to thermal stress on CuZnSOD activity. Dunn's test $(\alpha=0.05)$ was used for a posteriori comparisons.

\section{RESULTS}

Differences in the levels of CuZnSOD activity were found throughout the experiment (Kruskal-Wallis $H=$ 163.08, $P<0.001)$. These differences were determined by both the increase in temperature (Dunn's test, $P<0.05$ ) and the time of exposure to thermal stress (Dunn's test, $P<0.05$ ). The organisms in the $28^{\circ} \mathrm{C}$ treatment did not show significant changes in CuZnSOD activity. However, in the corals exposed to $31{ }^{\circ} \mathrm{C}, \mathrm{CuZnSOD}$ activity initially decreased from $0.311 \pm 0.16 \mathrm{U} \mathrm{mL}^{-1}(0 \mathrm{~h})$ to $0.144 \pm 0.02 \mathrm{U} \mathrm{mL}^{-1}$ at $18 \mathrm{~h}$, but then increased steadily until reaching $2.16 \pm 0.08 \mathrm{U} \mathrm{mL}^{-1}$ at $198 \mathrm{~h}$, which is nearly 10 times higher than the minimum value recorded (fig. 1).

\section{DISCUSSION}

Our findings show that an increase in temperature produced an increase in CuZnSOD activity and caused thermal stress leading to bleaching of the $P$. verrucosa fragments. By quantifying CuZnSOD activity, one of the initial oxidative stress responses is being evaluated, since $\mathrm{CuZnSOD}$ is one of the first proteins synthesized (Pal Yu 1994, Furla et al. 2005). Moreover, its synthesis triggers the production of antioxidants that will continue the reactions to eliminate ROS from the organism (cascade effect) (Logan et al. 1996). Since CuZnSOD activity only occurs in animals (Pal Yu 1994, duró 198 h, tiempo en el cual se observó que había iniciado el blanqueamiento.

Durante el periodo de aclimatación y el de experimentación, se tomaron registros de temperatura, salinidad y oxígeno disuelto cada hora. El oxígeno disuelto $\left(\mathrm{mg} \mathrm{L}^{-1}\right) \mathrm{se}$ midió con una sonda multiparamétrica (YSI 85). Independientemente, en cada acuario se colocó un termógrafo (HOBO Water Temp Pro) que registraba la temperatura del agua cada $15 \mathrm{~min}$.

Las condiciones de los acuarios se mantuvieron constantes durante el experimento. En el tratamiento a $28^{\circ} \mathrm{C}$ la temperatura se mantuvo en un intervalo de 27.74 a $28.52{ }^{\circ} \mathrm{C}$ con una media ( \pm devisación estandár) de $28.33 \pm 0.04^{\circ} \mathrm{C}$; la media de la salinidad fue $35.18 \pm 0.16$ y la del oxígeno disuelto, $5.88 \pm 0.07 \mathrm{mg} \mathrm{L}^{-1}$. En el tratamiento a $31{ }^{\circ} \mathrm{C}$ la temperatura varió entre 30.6 y $31.02^{\circ} \mathrm{C}$; la media de la salinidad fue de $35.24 \pm 0.21$ y la del oxígeno disuelto, $5.78 \pm$ $0.08 \mathrm{mg} \mathrm{L}^{-1}$.

\section{Cuantificación de la CuZnSOD}

La actividad de la CuZnSOD se determinó utilizando el kit SOD-525 de Bioxytech (Oxis International, Inc.). Para la obtención del tejido, las muestras preservadas en RNAlater fueron maceradas con $2.0 \mathrm{~mL}$ de una solución de $\mathrm{NaCl}$ al $0.9 \%$. Posteriormente, se tomaron $250 \mu \mathrm{L}$ del homogeneizado obtenido y se transfirieron a un tubo limpio, añadiéndole $400 \mu \mathrm{L}$ de una solución de etanol: cloroformo $(6.25: 37.50 \mathrm{v} / \mathrm{v})$. La muestra se agitó con un mezclador tipo vortex y se centrifugó a $3000 \mathrm{~g}$ durante $5 \mathrm{~min}$ a $4{ }^{\circ} \mathrm{C}$. Se esperaron de 3 a $5 \mathrm{~min}$, y la fase acuosa superior se transfirió a un tubo nuevo de $2.0 \mathrm{~mL}$. Este procedimiento inactiva a la $\mathrm{Mn}$ - y Fe-SOD, por lo que permite evaluar exclusivamente la actividad de la CuZnSOD.

El extracto fue tratado con el kit SOD-525 de Bioxytech de acuerdo con las instrucciones del proveedor, y se determinó la absorbancia a $525 \mathrm{~nm}$ en un espectofotómetro BioRad SmartSpec 3000. Se realizaron cuatro lecturas para estandarizar el blanco de acuerdo con las instrucciones del proveedor y se leyeron por duplicado las muestras del experimento. Finalmente, la actividad de la enzima fue expresada en unidades por mililitro ( $\mathrm{U} \mathrm{mL}^{-1} \pm$ error estándar).

Los análisis estadísticos fueron calculados utilizando el programa Sigma Plot v.11.0 para Windows. Se elaboró un análisis de Kruskal-Wallis (análisis de varianza de una vía por rangos, ya que no existió normalidad en los datos) para determinar el efecto de la temperatura y el tiempo de exposición al estrés térmico en la actividad de la CuZnSOD; se utilizó la prueba de Dunn $(\alpha=0.05)$ para comparaciones a posteriori.

\section{Resultados}

Los resultados mostraron que existieron diferencias en los niveles de actividad de la CuZnSOD a lo largo del 


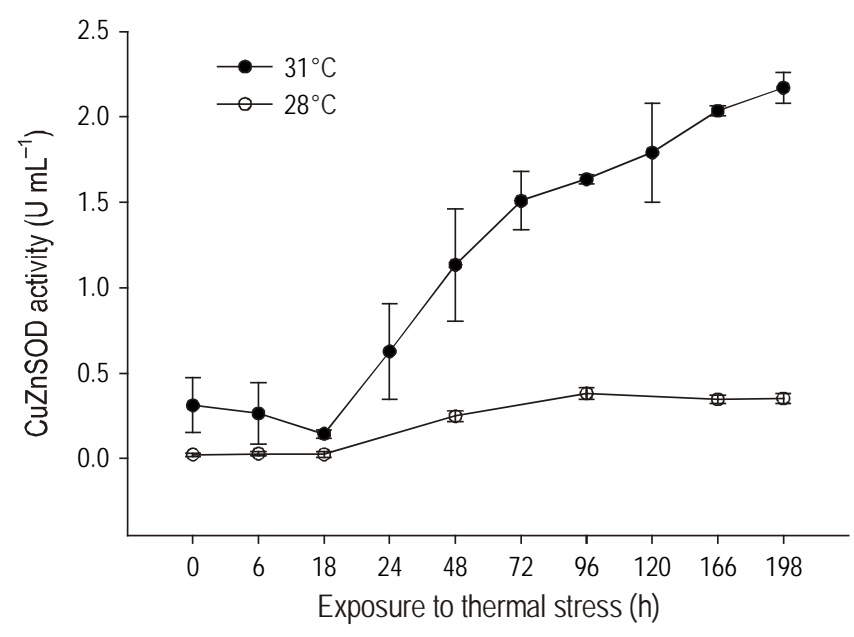

Figure 1. Enzymatic activity of CuZnSOD in fragments of Pocillopora verrucosa exposed to thermal stress conditions. Bars represent the standard error of the mean.

Figura 1. Actividad de la enzima CuZnSOD en fragmentos de Pocillopora verrucosa sometida a condiciones de estrés térmico. Las barras representan el error estándar de la media.

Logan et al. 1996, Zelko et al. 2002, Furla et al. 2005), the changes or increases in the levels of CuZnSOD activity are the host's response.

The increase in enzyme activity observed in the $31{ }^{\circ} \mathrm{C}$ treatment is the host's response triggered by the symbiont's stress response to increased temperature. An increase in temperature causes increased cellular metabolism (Hochachka and Somero 2002) and, as a result, greater ROS production. Since ROS affect cell structure and function, the organism activates antioxidant defense mechanisms to prevent cell damage (Lesser 2006). This is achieved by generating antioxidants such as SOD (Pal Yu 1994, Logan et al. 1996) that are broken down until non-toxic metabolites are obtained (Girotti 1998).

Our results for $P$. verrucosa concur with those obtained for other coral species (Downs et al. 2000, Yakovleva et al. 2004, Vidal-Dupiol et al. 2009). However, in Pocillopora capitata from the Mexican Pacific, Flores-Ramírez and Liñán-Cabello (2007) did not observe an increase in SOD activity due to thermal stress induced at a temperature of $32{ }^{\circ} \mathrm{C}$. The difference between the latter and our study can be attributed to the fact that the time of response can vary among species and even colonies from the same region (Yakovleva et al. 2004).

Coral communities in the Mexican Pacific are at the northern limit of their distribution (Glynn and Ault 2000). Thus, they can present regional differences in thermotolerance and response time due to local acclimation to suboptimal conditions for their development (Feder 1999, Olvier and Palumbi 2011) and their life history in general (Somero 2005, Middlebrook et al. 2008). Although annual temperature changes may not cause increased synthesis of SOD, as experimento $(H$ de Kruskal-Wallis $=163.08, P<0.001)$. Estas diferencias estuvieron determinadas por tanto el incremento en la temperatura (prueba de Dunn, $P<0.05$ ) como por el tiempo de estrés térmico (prueba de Dunn, $P<0.05$ ). Los organismos en el tratamiento a $28^{\circ} \mathrm{C}$ no presentaron cambios significativos en la actividad del antioxidante, mientras que los corales expuestos a $31{ }^{\circ} \mathrm{C}$ presentaron un decremento de $0.311 \pm 0.16 \mathrm{U} \mathrm{mL}^{-1}$ en la evaluación inicial a $0.144 \pm 0.02 \mathrm{U} \mathrm{mL}^{-1}$ a las $18 \mathrm{~h}$; sin embargo, a partir de la siguiente evaluación, se detectó un incremento continúo que alcanzó un máximo de $2.16 \pm 0.08 \mathrm{U} \mathrm{mL}^{-1}$ a las $198 \mathrm{~h}$, que es equivalente a casi 10 veces más el valor mínimo detectado (fig. 1).

\section{Discusión}

Los resultados evidencian que el incremento en la temperatura produjo un incremento en la actividad de la CuZnSOD y generó un estrés térmico que culminó en el blanqueamiento de los fragmentos de P. verrucosa. Al cuantificar la actividad de la CuZnSOD, se está evaluando uno de los eventos iniciales de respuesta del estrés oxidativo ya que la CuZnSOD es una de las primeras proteínas sintetizadas (Pal Yu 1994, Furla et al. 2005). Además, su síntesis genera como efecto cascada la producción de antioxidantes que seguirán las reacciones para eliminar otros ROS del organismo (Logan et al. 1996). Debido a que la CuZnSOD presenta actividad solamente en animales (Pal Yu 1994, Logan et al. 1996, Zelko et al. 2002, Furla et al. 2005), los cambios o aumentos en los niveles de la CuZnSOD son respuesta del hospedero.

Sin embargo, cabe señalar que el aumento en la actividad de la enzima registrado para el tratamiento a $31{ }^{\circ} \mathrm{C}$ es una respuesta del hospedero como consecuencia de la respuesta de estrés del simbionte ante el incremento en la temperatura. El incremento en la temperatura provoca un incremento metabólico a nivel celular (Hochachka y Somero 2002) y, como consecuencia, una mayor producción de ROS. Debido a que las ROS comprometen la estructura y función celular, el organismo activa mecanismos de defensa mediante agentes antioxidantes para prevenir el daño en sus células (Lesser 2006). Esto se logra con la generación de antioxidantes como la SOD (Pal Yu 1994, Logan et al. 1996), rompiéndolos hasta obtener metabolitos no tóxicos para el organismo (Girotti 1998).

Los resultados obtenidos para $P$. verrucosa en el presente estudio concuerdan con resultados obtenidos para otras especies de coral (Downs et al. 2000, Yakovleva et al. 2004, Vidal-Dupiol et al. 2009). Sin embargo, estudios previos sobre Pocillopora capitata, distribuida en el Pacífico mexicano, no mostraron un aumento en la actividad de la SOD por estrés térmico inducido a una temperatura de $32{ }^{\circ} \mathrm{C}$ (FloresRamírez y Liñán-Cabello 2007). Las diferencias entre este último y el presente estudio pueden deberse a que el tiempo de respuesta puede cambiar entre especies e incluso en colonias de la misma región (Yakovleva et al. 2004). 
has been recorded in regions near our study area (LiñánCabello et al. 2009), it is important to consider that other conditions, such as light intensity, in combination with thermal anomalies may induce SOD production (Downs et al. 2002, Saxby et al. 2003, Richier et al. 2008).

Rodríguez-Troncoso et al. (2010a, 2010b) used total lipid content and symbiont density as physiological markers to assess thermal stress in Pocillopora colonies from the central Mexican Pacific. The present study, however, examines thermal stress at the cellular level. We determined that CuZnSOD can be used as a complimentary biomarker in order to have, at both the physiological and cellular levels, tools to assess coral health and response under suboptimal temperature conditions before bleaching becomes evident.

\section{ACKNOWLEDGEMENTS}

The first author acknowledges receipt of a doctoral scholarship from the Mexican Council for Science and Technology (CONACYT No. 288773). This study was supported by the Autonomous University of Baja California (IIO-UABC internal project No. 533 to ECI) and by the University of Guadalajara (CUC-UG project to ACM). We thank the authorities of Marietas National Park for the facilities provided.

English translation by Christine Harris.

\section{REFERENCES}

Brown BE. 1997. Coral bleaching: Causes and consequences. Coral Reefs 16s: 129-138. http://dx.doi.org/10.1007/s003380050249

Downs CA, Mueller E, Phillips S, Fauth JE, Woodley CM. 2000. A molecular biomarker system for assessing the health of coral (Montastrea faveolata) during heat stress. Mar. Biotechnol. 2: 533-544. http://dx.doi.org/10.1007/s101260000038

Downs CA, Fauth JE, Halas JC, Dustan P, Bemiss J, Woodley CM. 2002. Oxidative stress and seasonal coral bleaching. Free Radic. Biol. Med. 33: 533-543. http://dx.doi.org/10.1016/S0891-5849(02)00907-3

Feder ME. 1999. Organismal, ecological, and evolutionary aspects of heat-shock proteins and the stress response: Established conclusions and un-resolved issues. Am. Zool. 39: 857-864. http://dx.doi.org/10.1093/icb/39.6.857

Fitt WK, Brown B, Warner ME, Dunne RP. 2001. Coral bleaching: Interpretation of thermal tolerance limits and thermal thresholds in tropical corals. Coral Reefs 20: 51-65. http://dx.doi.org/10.1007/s003380100146

Flores-Ramírez LA, Liñán-Cabello MA. 2007. Relationships among thermal stress, bleaching and oxidative damage in the hermatypic coral Pocillopora capitata. Comp. Biochem. Physiol. 146: 194-202. http://dx.doi.org/10.1016/j.cbpc.2006.09.008

Furla P, Allemand D, Shick JM, Ferrier-Pages C, Richier S, Plantivaux A, Allemand D. 2005. The symbiotic anthozoan: A physiological chimera between alga and animal. Integr. Comp. Biol. 45: 595-604. http://dx.doi.org/10.1093/icb/45.4.595
Las comunidades coralinas del Pacífico mexicano se encuentran localizadas en su límite norte de distribución (Glynn y Ault 2000). Por lo tanto, éstas pueden presentar diferencias regionales en la termotolerancia y el tiempo de respuesta debido a la aclimatación local a condiciones suboptimas para su desarrollo (Feder 1999, Olvier y Palumbi 2011) y, en su conjunto, su historia de vida (Somero 2005, Middlebrook et al. 2008). Aunque puede que los cambios anuales en la temperatura no produzcan una mayor síntesis de SOD, como ha sido registrado en regiones cercanas a nuestra área de estudio (Liñán-Cabello et al. 2009), sería importante considerar otras condiciones, como la intensidad lumínica, que en combinación con anomalías térmicas pueden inducir la producción de SOD (Downs et al. 2002, Saxby et al. 2003, Richier et al. 2008).

En estudios previos, Rodríguez-Troncoso et al. (2010a, b) utilizaron el contenido de lípidos totales y la densidad de simbiontes como marcadores físiológicos para evaluar el estrés térmico en los corales del Pacífico mexicano; sin embargo, en el presente estudio se evaluó el estrés térmico a nivel celular. Por lo tanto, la CuZnSOD podría considerarse como un posible biomarcador complementario para tener, tanto a nivel celular como fisiológico, herramientas para evaluar el estado de salud y la respuesta ante condiciones de temperatura subóptimas antes de que el blanqueamiento sea evidente durante los eventos de estrés térmico.

\section{Agradecimientos}

El primer autor recibió apoyo del Consejo Nacional de Ciencia y Tecnología (CONACYT, beca de doctorado No. 288773). El presente estudio fue financiado por la Universidad Autónoma de Baja California (IIO-UABC, proyecto No. 533 a cargo de ECI) y la Universidad de Guadalajara (CUCUG, proyecto a cargo de ACM). Los autores agradecen a las autoridades del Parque Nacional Islas Marietas por las facilidades otorgadas para el muestreo.

Gates RD, Edmunds PJ. 1999. The physiological mechanisms of acclimatization in tropical reef corals. Am. Zool. 39: 30-43. http://dx.doi.org/10.1093/icb/39.1.30

Girotti AW. 1998. Lipid hydroperoxide generation, turnover and effector action in biological systems. J. Lipid Res. 39: 1529-1542.

Glynn PW, Ault JS. 2000. A biogeographic analysis and review of the far eastern Pacific coral reef region. Coral Reefs 19: 1-23. http://dx.doi.org/10.1007/s003380050220

Hochachka PW, Somero GN. 2002. Biochemical Adaptation. Oxford University Press, Oxford, 466 pp.

Hoegh-Guldberg O. 1999. Climate change, coral bleaching and the future of the world's coral reefs. Mar. Freshwat. Res. 50: 839-866. http://dx.doi.org/10.1071/MF99078

Hoegh-Guldberg O, Mumby PJ, Hooten AJ, Steneck RS, Greenfield P, Gomez E, Harvell CD, Sale PF, Edwards AJ, Caldeira K, Knowlton N, Eakin CM, Iglesias-Prieto R, Muthiga N, Dubi A, 
Hatziolos ME. 2007. Coral reefs under rapid climate change and ocean acidification. Science 318: 1737-1742. http://dx.doi.org/10.1126/science.1152509

Kleypas JA, Buddemeir RW, Gattuso JP. 2001. The future of coral reefs in an age of global change. Int. J. Earth Sci. 90: 426-437. http://dx.doi.org/10.1007/s005310000125

Lesser MP. 2006. Oxidative stress in marine environments: Biochemistry and physiological ecology. Annu. Rev. Physiol. 68: 253-278.

http://dx.doi.org/10.1146/annurev.physiol.68.040104.110001

Liñán-Cabello MA, Flores-Ramírez LA, Cobo-Díaz JF, ZentenoSavin T, Olguín-Monroy O, Sosa-Avalos R, Patiño-Barragan M, Olivos-Ortiz A. 2009. Seasonal changes of antioxidant and oxidative parameters in the coral Pocillopora capitata on the Pacific coast of Mexico. Mar. Ecol. 31: 407-417. http://dx.doi.org/10.1111/j.1439-0485.2009.00349.x

Logan BA, Kornyeyev D, Hardison J, Scott AH. 1996. The role of antioxidant enzymes in photoprotection. Photosynth. Res. 88: 119-132. http://dx.doi.org/10.1007/s11120-006-9043-2

Middlebrook R, Hoegh-Guldberg O, Leggat W. 2008. The effect of thermal history on the susceptibility of reef-building corals to thermal stress. J. Exp. Biol. 211: 1050-1056. http://dx.doi.org/10.1242/jeb.013284

Oliver TA, Palumbi SR. 2011. Do fluctuating temperature environments elevate coral thermal tolerance? Coral Reefs 30: 429-440. http://dx.doi.org/10.1007/s00338-011-0721-y

Pal Yu B. 1994. Cellular defenses against damage from reactive species. Physiol. Rev. 74: 139-162.

Richier S, Furla P, Plantivaux A, Merle PL, Allemand D. 2003. Symbiosis-induced adaptation to oxidative stress. J. Exp. Biol. 208: 277-285. http://dx.doi.org/10.1242/jeb.01368

Richier S, Cottalorda JM, Guillaume M, Fernandez C, Allemand D, Furla P. 2008. Depth-dependant response to light of the reef building coral, Pocillopora verrucosa: Implication of oxidative stress. J. Exp. Mar. Biol. Ecol. 357: 48-56. http://dx.doi.org/10.1016/j.jembe.2007.12.026

Rodríguez-Troncoso AP, Carpizo-Ituarte E, Cupul-Magana AL. 2010a. Differential response to cold and warm water conditions in Pocillopora colonies from the Central Mexican Pacific.
J. Exp. Mar. Biol. Ecol. 391: 57-64. http://dx.doi.org/10.1016/j.jembe.2010.06.006

Rodríguez-Troncoso AP, Carpizo-Ituarte E, Cupul-Magaña A. 2010b. Response to sudden increases in temperature (thermal shock) under laboratory conditions in the coral Pocillopora verrucosa. Cienc. Mar. 36: 91-98.

Saxby T, Dennison WC, Hoegh-Gulberg O. 2003. Photosynthetic responses of the coral Montiopora digitata to cold temperature stress. Mar. Ecol. Prog. Ser. 248: 85-97. http://dx.doi.org/10.3354/meps248085

Somero GN. 2005. Linking biogeography to physiology: Evolutionary and acclimatory adjustments of thermal limits. Front. Zool. 2: 1-9. http://dx.doi.org/10.1186/1742-9994-2-1

Van Oppen MJH, Gates RD. 2006. Conservation genetics and the resilience of reef-building corals. Mol. Ecol. 15: 3863-3883. http://dx.doi.org/10.1111/j.1365-294X.2006.03026.x

Veron JEN, Hoegh-Guldberg O, Lenton TM, Lough JM, Obura DO, Pearce-Kelly P, Sheppard CRC, Spalding M, Stafford-Smith MG, Rogers AD. 2009. The coral reef crisis: The critical importance of $<350$ ppm $\mathrm{CO}_{2}$. Mar. Pollut. Bull. 58: $1428-1436$. http://dx.doi.org/10.1016/j.marpolbul.2009.09.009

Vidal-Dupiol J, Adjeroud M, Roger E, Foure L, Duval D, Mone Y, Ferrier-Pages C, Tambutte E, Tambutte S, Zoccola D, Allemand D, Mitta G. 2009. Coral bleaching under thermal stress: Putative involvement of host/symbiont recognition mechanisms. BMC Physiol. 9: 14. http://dx.doi.org/10.1186/1472-6793-9-14

Weis VM. 2008. Cellular mechanisms of cnidarian bleaching: stress causes the collapse of symbiosis. J. Exp. Biol. 211: 3059-3066. http://dx.doi.org/10.1242/jeb.009597

Yakovleva I, Bhagooli R, Takemura A, Hidaka M. 2004. Differential susceptibility to oxidative stress of two scleractinian corals: antioxidant functioning of mycrosporine-glycine. Comp. Biochem. Physiol. B 139: 721-730. http://dx.doi.org/10.1016/j.cbpc.2004.08.016

Zelko IN, Mariani TJ, Folz RJ. 2002. Superoxide dismutase multigene family: a comparison of the CuZn-SOD (SOD1), MnSOD (SOD2), and EC-SOD (SOD3) gene structures, evolution and expression. Free Radic. Biol. Med. 33: 337-349. http://dx.doi.org/10.1016/S0891-5849(02)00905-X

Received August 2012, received in revised form January 2013, accepted January 2013. 